\title{
COMBATE QUÍMICO DE MALEZAS EN MANZANO (Pyrus malus L.): COADYUVANTES 1
}

\author{
José Juan Cerda², Mariano Mendoza², Josafad Santiago ${ }^{2}$, Francisco Nieto ${ }^{2}$, Sergio Cortez ${ }^{2}$
}

\section{RESUMEN}

Combate químico de malezas en manzano (Pyrus malus L.): coadyuvantes. El objetivo de este trabajo fue determinar la eficiencia de los herbicidas paraquat y glifosato con cuatro coadyuvantes en el control postemergente de malezas; así como su factibilidad económica en relación al método mecánico . El estudio se realizó en la Sierra de Arteaga, Coahuila, México. Se tomaron datos a los 3, 13, 17, 31 y 83 días después de la aplicación. Se utilizó un diseño bloques al azar con cuatro repeticiones. La mezcla del glifosato + adherente controló un $80 \%$ de malezas hasta los 83 días después de la aplicación, el glifosato + humectante y el glifosato + ácido húmico brindaron un control del 78,6 y 77,1\% respectivamente. El paraquat con el ácido húmico controló un $88,4 \%$ hasta los 31 días y el paraquat + adherente tuvo un $75,1 \%$ de control. En general, se observó que el glifosato con los coadyuvantes mostró mejor control hasta los 83 días. El valor económico de dicho tratamiento fue de $\$ 346,3 \mathrm{MN}$, mientras que el método mecánico costó $\$ 810,0 \mathrm{MN}$ y se consideró el método químico más rentable.

\begin{abstract}
Weed chemical control in apple (Pyrus malus L.): coadjutants. This study was carried out in order to determine the efficiency of paraquat and glyphosate herbicides mixed with four coadjutants for post-emergent weed control, as compared to mechanical control, as well as their economic feasibility. The study took place in Sierra de Arteaga, Coahuila, México. Information was collected 3, 13, 17, 31, and 83 days after application. A randomized block design with four repetitions was used. Glyphosato combined with adherent controlled $80 \%$ of weeds 83 days after application; glyphosate combined with moisturizer, and glyphosate combined with humic acid yielded 78.6 and $77.1 \%$ control respectively. Parquat mixed with humic acid controlled $88.4 \% 31$ days after application and parquat mixed with adherent showed $75.1 \%$ control after 31 days. Overall, glyphosato combined with coadjutants showed better weed control 83 days after application. The cost of such treatment was $\$ 346.3$ (Mexican currency), while the cost for the mechanical method was $\$ 810.00$ (Mexican currency). The chemical method proved to be the most cost-efficient.
\end{abstract}

ducción regional en promedio es baja, tanto en riego ( siete $\mathrm{t} / \mathrm{ha}$ ) como en temporal (2,5 t/ha); sin embargo, es posible obtener producciones de 30 t/ha o más al eliminar aquellos factores que limitan la producción de acuerdo al área en que se encuentra localizada la huerta (Ramírez y Cepeda, 1993).

Entre la problemática a considerar, la fitosanidad es un factor determinante para el buen rendimiento y calidad de la cosecha, en particular cuando se refiere a malezas, ya que causan daños directos o indirectos en las plantaciones de dicho frutal, originando finalmente un resultado desfavorable para el productor. Las malezas, tanto anuales como perennes presentan ciertas características anatómicas y morfológicas que determinan

1 Presentado en la XLIII Reunión Anual del PCCMCA, Nicaragua, 1998.

2 Universidad Autónoma Agraria “Antonio Narro”. Buenavista, Saltillo, Coah, Méx. Tel/fax(84) 17-73-61. E-mail: postfito@uaaan.mx 
la calidad y el lapso de tiempo en que un herbicida ha de ser retenido y absorbido. Las anuales son controladas eficazmente con algún herbicida de contacto; en cambio, los herbicidas sistémicos resultan muy ventajosos contra las malezas perennes ya establecidas. En algunas ocasiones conviene emplear mezclas de dos o más productos químicos con el fin de combatir varias especies de malezas con una sola aplicación, buscando siempre que sea posible un efecto sinergísta, lo cual aumenta la eficiencia, ahorro del producto y disminución en el costo. Una razón de mezclar un surfactante con el herbicida, es que se logra mayor penetración de este último y los síntomas son más rápidos.

Por los antecedentes mencionados anteriormente el objetivo del presente fue determinar la eficiencia para el control postemergente de malezas en manzano de los herbicidas paraquat y glifosato, en combinación con cuatro coadyuvantes (adherente, ácido húmico, humectante y extensor) y determinar la factibilidad económica de los tratamientos evaluados.

Las malezas que infestan a los cultivos representan uno de los principales problemas al grado que ha sido estimado en forma general, que las pérdidas globales que ocasionan a la agricultura (42\%) son mayores que las ocasionadas por plagas insectiles (28\%), enfermedades (27\%) y nemátodos (3\%) (Tamayo, 1990).

El descuido del control de malezas en manzano durante el primer año puede reducir el periodo de crecimiento por más del 60 por ciento durante el periodo de competición. Los cultivos establecidos son también susceptibles a la competición; esto se demostró en un experimento con una densidad de cinco malezas $/ \mathrm{m}^{2}$, las que causaron un $30 \%$ de reducción en el crecimiento de árboles de manzano de dos años de edad (Roberts, 1982).

El valor comercial de la producción de manzana es determinado principalmente por el tamaño de la fruta, el cual se ve reducido por efecto de las malezas (Roberts, 1982).

En muchas ocasiones, los posibles efectos benéficos de las malas hierbas se ven opacados debido a que su presencia puede ser perjudicial al convertirse en focos de contaminación o diseminación de enfermedades y plagas que atacan a los árboles frutales (Calderón, 1983).

El polocote Helianthus malus es el principal hospedero de la araña roja (Tetranychus telarius) durante el invierno; ya sea en estado de huevo o adulto. Posteriormente esta plaga pasa al manzano (Sierra, 1991). La eliminación de malezas en el cajete del árbol (área del contorno del árbol donde se aplica el riego y otros insu- mos útiles al mismo) de manzano, reduce la presencia del picudo negro (Amphidees major y A. macer) en éste y por consiguiente, el daño (Ramírez y Cepeda, 1993).

Una cobertura de malezas permanente que llegue hasta el pie del árbol de manzano, favorece una constante y alta humedad en la zona del cuello, lo que beneficia al hongo Phytophthora cactarum, el cual es causante de la podredumbre del cuello de dicho frutal; y a la bacteria Agrobacterium tumefasciens, que ocasiona el agallamiento en la corona del árbol. Por consiguiente es necesario segarlo, en caso de usar herbicidas, emplearlos en un radio de 30 cm o más del pie del árbol (Durán, 1976).

La eliminación de la maleza en las plantaciones de manzano es conveniente realizarla antes de plantar los árboles (Kramer, et al., 1982).

Existen diferentes métodos opcionales para realizar un control satisfactorio de malezas en manzano, entre éstos se encuentra el preventivo, legal, manual, mecánico, cultural, físico, biológico integrado y químico (Arroyo, 1980; Rojas, 1990 y NAS, 1990), pero el control químico es hoy en día la alternativa más viable (Morgado, 1990).

El uso de surfactantes y agentes humectantes adecuados aumentan la absorción tanto a través de los estomas como a través de la cutícula, alterando la selectividad (SOMECIMA, 1986; Klingman y Ashton, 1991)

Una forma de lograr mayor penetración de los herbicidas, ha sido adicionando coadyuvantes a las formulaciones, o bien mezclando éstos al momento de la aplicación. Los surfactantes pueden aumentar la toxicidad provocada por el herbicida a la maleza, pero también pueden ser más tóxicos para el cultivo. Aplicados al suelo se usan para incrementar la infiltración de mezclas de productos químicos y agua (Medina, 1983; Urzúa y Sánchez, 1991).

La acción de un herbicida comprende tres etapas: la penetración en la planta, la translocación del herbicida desde el punto de penetración hasta el sitio de la función fisiológica sensible y el modo de acción propiamente dicho, es decir, el efecto del herbicida sobre los procesos bioquímicos celulares y las consecuencias fisiológicas que de ahí se derivan (Tamayo, 1988).

El glifosato es un herbicida alifático sistémico, derivado de los picolínicos, no selectivo que puede aplicarse en pre-siembra o post-emergencia; teniendo también uso urbano (SOMECIMA, 1986; "CICOPLAFEST", 1991). El paraquat es un herbicida que pertenece al grupo de los bipiridilos y se usa como herbici- 
da no selectivo de contacto post-emergente (SOMECIMA,1986; “CICOPLAFEST”, 1991).

El glifosato a razón de 0,$56 ; 1,12$ y 2,24 kg/ha con 0,5 porciento (volumen/volumen) de coadyuvante, incrementó el control sobre zacates y plantas de hoja ancha Young (1984).

Además el glifosato a dosis de $0,4 \mathrm{~kg} / \mathrm{ha}$ en mezcla con agral plus a razón de 0,25 porciento, incrementó su control sobre Brachiaria plantaginea, Cynodon dactylon, Sporobolus sp, Mullembergia spp y Cyperus rotundus Bolaños y Rosas (1991). Urzúa y Sánchez (1991) demostraron que el paraquat a dosis de $0,15 \mathrm{~kg} / \mathrm{ha}$ en combinación con agral plus a razón de 0,25 porciento (volumen/volumen), incremento su acción sobre "aceitilla" (Bidens odorata) y "quelite" (Amaranthus hybridus) de 10 a 15 días de emergidos.

\section{MATERIALES Y MÉTODOS}

El presente trabajo se desarrolló en la región manzanera de la Sierra de Arteaga, ubicada al sureste del estado de Coahuila, México a $25^{\circ} 31^{\prime}$ Latitud Norte y $100^{\circ} 41^{\prime}$ Latitud Oeste y una altitud estimada de 2200 msnm. El clima corresponde al templado sub-húmedo con veranos cálidos e inviernos benignos, con una precipitación pluvial anual de $600 \mathrm{~mm}$ y dos épocas de sequía. El tipo de suelo pertenece a migajón arcilloso; posee una acumulación de calcio con textura fina, tiene un $32 \%$ de arcilla, un $28 \%$ de limo y un $40 \%$ de arena; son permeables y petrocálcicos con una profundidad de 30 cm cuya limitante es la cimentación. El sistema de plantación utilizado fue el rectangular con una distancia entre surcos de $8 \times 6 \mathrm{~m}$.
La investigación inició en 1993 en manzano variedad Red Delicious. Se realizó una sola aplicación de los diferentes tratamientos en el cajete del árbol, calle e hilera de la plantación, en árboles de cinco años de edad bajo condiciones de riego, con una fertilización óptima del suelo. Se utilizó como unidad experimental una superficie de $16 \mathrm{~m}^{2}$, distribuida en cuatro repeticiones, los datos se analizaron con un diseño de bloques al azar.

La aplicación se realizó sobre la maleza establecida, la cual presentó una altura promedio de $17 \mathrm{~cm}$, empleándose para tal efecto herbicidas post-emergentes en mezcla individual con cuatro coadyuvantes a las dosis comerciales (Cuadro 1) en una superficie total de $352 \mathrm{~m}^{2}$, donde fueron distribuidos los 11 tratamientos, entre los que se incluye un testigo sin la aplicación del herbicida (TSAH).

La aplicación de los tratamientos se hizo con una aspersora manual de presión constante a base de $\mathrm{CO}_{2}$ tipo "Weed System Sprayer" y boquillas Tee Jeet 8002 , calibrada para un gasto de 3001 de agua por hectárea a una presión de 35 libras por pulgada cuadrada (psi).

La toma de datos se realizó a las tres, 13, 17, 31 y 83 días después de la aplicación de los tratamientos (DDA). Los parámetros evaluados mediante muestreos cuantitativos fueron:

(1) Control (\%) de la maleza mediante conteos de la mortalidad observada en cada tratamiento herbicida, comparándola con el testigo (TSAH).

(2) Fitotoxicidad foliar y sintomatología general que presento la maleza, representando el grado de fitotoxicidad de acuerdo a la escala EWRC (Cuadro 2).

Cuadro 1. Tratamientos evaluados para el control postemergente de malezas en manzano. Arteaga, Coahuila. 1994.

\begin{tabular}{lllc}
\hline $\begin{array}{l}\text { Tratamiento } \\
\text { Nombre técnico }\end{array}$ & $\begin{array}{c}\text { Dosis }(\mathbf{g}) * \\
\text { Ingrediente activo/ha }\end{array}$ & $\begin{array}{c}\text { Tratamiento } \\
\text { Nombre comercial }\end{array}$ & $\begin{array}{c}\text { Dosis (l+ ml) } \\
\text { Material comercial }\end{array}$ \\
\hline Paraquat & 400 & Gramoxone & 2,0 \\
Paraquat + adherente & $300+480$ & Faena + Frigate & $1,5+600$ \\
Paraquat + Acido Húmico & $300+90$ & Gramoxone + Humicel & $1,5+750$ \\
Paraquat + Humectante & $300+182$ & Gramoxone + Bionex & $1,5+600$ \\
Paraquat + Extensor & $300+180$ & Gramoxone + Agral Plus & $1,5+600$ \\
Glifosato & 1920 & Faena & 4,0 \\
Glifosato + Adherente & $1440+240$ & Faena + Frigate & $3,0+300$ \\
Glifosato + Acido Húmico & $1440+72$ & Faena + Humicel & $3,0+600$ \\
Glifosato + Humectante & $1440+137$ & Faena + Bionex & $3,0+450$ \\
Glifosato + Extensor & $1440+90$ & Faena + Agral Plus & $3,0+300$ \\
TSAH & Testigo & Testigo & Testigo \\
\hline
\end{tabular}


Cuadro 2. Escala EWRC (Europen Weed Research Council) adaptada y modificada para evaluaciones visuales del comportamiento de herbicidas en el control de malezas. 1994.

\begin{tabular}{llll}
\hline \multicolumn{5}{c}{ Efecto sobre la maleza } \\
\hline Grado & Muertes (\%) & Grado & \multicolumn{1}{c}{ Fitotoxicidad (\%) } \\
\hline 0 & No efecto aparente & 0 & No efecto aparente \\
1 & 1 a 20 & 2 & 1 a 20 plantas dañadas \\
2 & 20 a 29 & 4 & 21 a 40 plantas dañadas \\
3 & 30 a 39 & 6 & 41 a 60 plantas dañadas \\
4 & 40 a 49 & 8 & 61 a 80 plantas dañadas \\
5 & 50 a 59 & 10 & 81 a 100 plantas dañadas \\
6 & 60 a 69 & & \\
7 & 70 a 79 & 0 & No efecto aparente \\
8 & 80 a 89 & 1 & Daño mínimo \\
9 & 90 a 100 & 2 & Daño leve \\
& & 3 & Daño regular \\
& & 4 & Daño severo \\
& & 5 & Daño muy severo \\
\hline
\end{tabular}

El efecto fitotóxico sobre las malas hierbas a las cuales se les hizo la aplicación de los tratamiento, se cuantificó según escala para grado de muerte, grado de fitotoxicidad y tipo de daño, las cuales se basaron en la escala de EWRC (Cuadro 2).

Para determinar el efecto fitotóxico de los tratamientos en las malas hierbas, las observaciones se hicieron en cada una de las cuatro repeticiones de cada tratamiento, considerando un $\mathrm{m}^{2}$ por parcela. $\mathrm{Pa}-$ ra determinar la diversidad de especies de malezas y conocer la (s) predominante (s), así como su altura promedio $(\mathrm{cm})$, número de individuos promedio por especie por parcela; se tomaron datos de 10 parcelas, las cuales presentaban una densidad de población elevada $(80-90 \%)$.

Los resultados fueron analizados estadísticamente a través del diseño de bloques al azar con 11 tratamientos y cuatro repeticiones. Al detectar significancia entre tratamientos en los análisis de varianza se realizó la comparación de medias, mediante Tukey al $1 \%$.

\section{RESULTADOS Y DISCUSIÓN}

Mediante muestreos realizados en el testigo sin la aplicación del herbicida (TSAH), se determinó que las principales especies de malezas que infestan al cultivo de manzano en la región del Cañon de los Lirios, Arteaga, Coahuila, (Cuadro 3) fueron de acuerdo a su densidad poblacional, el polocotillo Helianthus laciniatus
(33\%) y la Correhuela Ipomea purpurea (25\%). En menor grado de infestación se encontraron presentes la Hierba del golpe Gaura coccinea (12\%), Quelite cenizo Chenopodium album (10\%), Fumaria parviflora (8\%), Zacate salvación Bromus unioloides (7\%) y Alfilerillo Erodium cicutarium (5\%).

De acuerdo a lo anterior, la infestación estuvo compuesta de malezas anuales como "correhuela", "quelite cenizo", "fumaria", zacate salvación y alfilerillo, con una infestación global del $55 \%$ y malezas perennes como el polocotillo y hierba del golpe con una infestación del $45 \%$.

Considerando la dominancia de malezas perennes y dado que el control de estas es mas efectivo por medios químicos, en base al Cuadro 4, se puede deducir los mejores tratamientos, al menos tres días después de la aplicación. En dicho cuadro, de acuerdo a la escala EWRC no se presentaron muertes; sin embargo, hubo una fitotoxicidad del 20 al 40 porciento en la población de maleza, sobresaliendo los tratamientos paraquat en mezcla con adherente o con extensor que provocaron una fitotoxicidad del $40 \%$ y daño severo; daño severo y un daño regular con humectante y $40 \%$ de fitotoxicidad. En este herbicida se vió el efecto fitotóxico de una manera rápida, observándose en general un daño regular, cuyos síntomas fueron secamientos en la parte de la hoja donde se asperjó el producto. En las malezas de hoja ancha como el polocotillo, correhuela, hierba del golpe y quelite cenizo se tuvo un efecto del $50 \%$ del área foliar, mientras que en fumaria y el alfilerillo, al igual que en la hoja angosta del zacate salvación un $80 \%$, esta diferencia se debió a la cobertura de cada especie, evitando o facilitando la aspersión sobre la anatomía y morfología de la planta.

Cabe señalar que el efecto del paraquat, siendo un herbicida de contacto, se manifiesta a partir del primer día después de la aplicación, no así para el glifosato que es sistémico y cuyo daño se observa hasta una semana después de dicha aplicación, por esta razón el daño detectado sobre el follaje de la maleza fue mínimo, el cual consistió de un ligero marchitamiento en la parte superior del follaje de todas y cada una de las diferentes especies de malezas presentes al momento de la aplicación, además de una ligera clorosis. No obstante cuando se usó adherente el glifosato mostró mayor fitotoxicidad inicial, $40 \%$ al tercer día.

A los 13 días después de la aplicación (Cuadro 5), el daño del paraquat puede considerarse máximo de acuerdo a su tipo de acción, y el efecto del glifosato está en plena manifestación; así, el tratamiento del paraquat + adherente y el glifosato + humectante son los 
Cuadro 3. Diversidad, densidad poblacional y altura de malas hierbas presentes al momento de la aplicación en el cultivo de manzano en el Cañon de los Lirios, Arteaga, Coahuila. 1994.

\begin{tabular}{lllcrrr}
\hline Nombre Científico & \multicolumn{1}{c}{$\begin{array}{c}\text { Nombre } \\
\text { común }(\mathbf{c m})\end{array}$} & $\begin{array}{c}\text { Familia Taxo- } \\
\text { nómica }\left(\mathbf{m}^{\mathbf{2}}\right)\end{array}$ & $\begin{array}{c}\text { Altura } \\
\text { Media (ha) }\end{array}$ & $\begin{array}{c}\mathbf{N}^{\mathbf{0}} \text { de } \\
\text { Individuos }\end{array}$ & $\begin{array}{c}\mathbf{N}^{\mathbf{0}} \text { de } \\
\text { Individuos }\end{array}$ & $\begin{array}{c}\text { Infestación } \\
(\%)\end{array}$ \\
\hline Helianthus laciniatus Gray & Polocotillo & Asteraceae & 23 & 29 & 290000 & 33 \\
Ipomea purpurea (L) Roth & Correhuela & Convulvolaceae & 12 & 22 & 220000 & 25 \\
Gaura coccinia Push & Hierba del golpe & Onagraceae & 17 & 10 & 100000 & 12 \\
Chenopodium album L & Quelite cenizo & Chenopodiaceae & 11 & 9 & 90000 & 10 \\
Fumaria parviflora Lam. & Fumaria & Fumariaceae & 15 & 7 & 70000 & 8 \\
Bromus unioloides H.B.K & Zacate salvación & Poaceae & 19 & 6 & 60000 & 7 \\
Erodium cicutarium (L) L'Her. & Alfilerillo & Geraniaceae & 11 & 4 & 40000 & 5 \\
& & & Total & $\mathbf{8 7}$ & $\mathbf{8 7 0 ~ 0 0 0}$ & $\mathbf{1 0 0}$ \\
\hline
\end{tabular}

Cuadro 4. Efecto sobre las malezas citadas en el Cuadro 3 con los herbicidas postemergentes glifosato y paraquat en mezcla con diferentes surfactantes, 3 DDA en manzano. Arteaga. Coahuila. 1994.

\begin{tabular}{lcccl}
\hline $\begin{array}{l}\text { Tratamiento } \\
\text { Nombre técnico }\end{array}$ & $\begin{array}{c}\text { Dosis }(\mathbf{g}) \\
\text { Ingrediente activo/ha }\end{array}$ & $\begin{array}{c}\text { Esacala EWRC } \\
\text { Muertes (\%) }\end{array}$ & $\begin{array}{c}\text { Daño } \\
\text { Fitotoxicidad (\%) }\end{array}$ & \begin{tabular}{c} 
observado \\
\hline Paraquat
\end{tabular} \\
Paraquat + Adherente & 300 & 0 & $20^{*}$ & Leve \\
Paraquat + Acido Húmico & $300+480$ & 0 & 40 & Severo \\
Paraquat + Humectante & $300+182$ & 0 & 20 & Leve \\
Paraquat + Extensor & $300+180$ & 0 & 40 & Regular \\
Glifosato & 1920 & 0 & 40 & Severo \\
Glifosato + Adherente & $1440+240$ & 0 & 20 & Leve \\
Glifosato + Acido Húmico & $1440+72$ & 0 & 40 & Mínimo \\
Glifosato + Humectante & $1440+137$ & 0 & 20 & Mínimo \\
Glifosato + Extensor & $1440+90$ & 0 & 20 & Mínimo \\
TSAH & Testigo & 0 & 20 & Mínimo \\
\hline
\end{tabular}

* El porcentaje restante no presentó efecto aparente.

Cuadro 5. Efecto sobre maleza de los herbicidas postemergentes glifosato y paraquat en mezcla con diferentes surfactantes, 13 DDA en manzano. Arteaga. Coahuila. 1994.

\begin{tabular}{lcccc}
\hline $\begin{array}{l}\text { Tratamiento } \\
\text { Nombre técnico }\end{array}$ & $\begin{array}{c}\text { Dosis }(\mathbf{g}) \\
\text { Ingrediente activo/ha }\end{array}$ & Muertes (\%) & $\begin{array}{c}\text { Escala EWRC } \\
\text { Fitotoxicidad (\%) }\end{array}$ & $\begin{array}{c}\text { Daño } \\
\text { observado }\end{array}$ \\
\hline Paraquat & 400 & 10 & $20 *$ & Leve \\
Paraquat + adherente & $300+480$ & 30 & 60 & Severo \\
Paraquat + Acido Húmico & $300+90$ & 10 & 60 & Leve \\
Paraquat + Humectante & $300+182$ & 10 & 60 & Regular \\
Paraquat + Extensor & $300+180$ & 20 & 40 & Severo \\
Glifosato & 1920 & 10 & 20 & Leve \\
Glifosato + Adherente & $1440+240$ & 10 & 40 & Mínimo \\
Glifosato + Acido Húmico & $1440+72$ & 10 & 60 & Mínimo \\
Glifosato + Humectante & $1440+137$ & 30 & 60 & Mínimo \\
Glifosato + Extensor & $1440+90$ & 10 & 40 & Mínimo \\
TSAH & Testigo & 0 & 0 & No aparente \\
\hline
\end{tabular}

* El porcentaje restante no presentó efecto aparente 
más sobresalientes, causando muertes del 30 porciento, una fitotoxicidad del 60 porciento y un tipo de daño muy severo y severo respectivamente, por lo que tal efecto se puede considerar como un $90 \%$ de control.

Del resto de los tratamientos, el paraquat + humectante y el paraquat + extensor sobresalieron, ya que ocasionaron daños muy severos sobre el follaje, controlando en un 70 y 60 porciento respectivamente. Otras mezclas como el paraquat + ácido húmico y el glifosato + ácido húmico causaron un daño y control regular; en las combinaciones restantes se observó un daño regular, asumiendo un control inferior al $50 \%$.

El comportamiento de los tratamientos presentó cambios radicales al evaluarse los datos tomados a los 17 días después de la aplicación (Cuadro 6), siendo procesados estadísticamente. Aunque no presentaron diferencias estadísticas significativa entre ellos, los porcentajes de control observados son significativamente diferentes a nivel de campo los porcentajes de control fueron diferentes; destacando en primer lugar la mezcla de glifosato + adherente a dosis de $1440 \mathrm{~g}+240$ $\mathrm{ml}$, pues fue el que mejor controló (85\%) a la maleza presente, por lo que se consideró satisfactorio su efecto; después le siguió el glifosato + extensor y el glifosato + ácido húmico, con un 80 y 79,7 porciento de control respectivamente. Del resto de tratamientos, el glifosato + humectante $(78,4 \%)$, paraquat + extensor $(75,4 \%)$ y el paraquat + humectante $(70,0 \%)$ se les considera tratamientos aceptables para el control eficiente de malezas (superior al $70 \%$ de control).

Si los 17 DDA el glifosato con sus respectivos aditivos (adherente, extensor, ácido húmico y humectante) sobresalió por su mayor control, a pesar de que todavía no expresaba su máxima capacidad fitotóxica para dañar a las plantas nocivas, en cambio, el paraquat solamente en mezcla individual con dos surfactantes (adherente y ácido húmico), entró a la selección de los productos considerados como aceptables.

A los 31 días de la aplicación, el glifosato + adherente montró la mayor efectividad ya que el $100 \%$ de las malezas aplicadas con este tratamiento habían muerto. Similarmente el glifosato + humectante y el glifosato + ácido húmico estadísticamente no diferentes, controlaron adecuadamente a la maleza, puesto que suprimieron el 98,5 y 98 porciento respectivamente de su población (Cuadro 7). El glifosato + extensor, se ubicó en cuarto lugar con un control de 95,3 porciento, comparativamente glifosato sin aditivos sólo controló un $70 \%$ de las malezas; indicándose así el efecto positivo de usar aditivos. El paraquat + ácido húmico y el paraquat + humectante se colocaron entre los aceptables, superando incluso al paraquat + humectante que anteriormente era el último y que actualmente no incrementó mucho su control (solamente $0,9 \%$ ), en cambio, las mezclas antes mencionadas aumentaron su porcentaje de control a 88,4 y 75,1 respectivamente. Otro de los tratamientos que se colocó dentro de los clasificados con buenos resultados, es el glifosato en su aplicación individual, presentando un control del 70,1\%. Los otros dos tratamientos restantes, entre los que se encuentran el paraquat + extensor que bajó notablemente su capacidad fitotóxica (a 69,9\%) puesto que en el Cuadro 6, se le considera como bueno, ahora al igual que el paraquat en su presentación individual, no son adecuados para un control óptimo de malezas. Cabe señalar que en este cuadro tampoco se encontró diferencia estadística entre tratamientos, sin embargo, el comportamiento en campo marcó grandes diferencias al momento de la limpieza del terreno.

Cuadro 6. Control de malezas en manzano con glifosato y paraquat en mezcla con diferentes surfactantes, 17 DDA en manzano. Arteaga. Coahuila. 1994.

\begin{tabular}{lccc}
\hline $\begin{array}{l}\text { Tratamiento } \\
\text { Nombre técnico }\end{array}$ & $\begin{array}{c}\text { Dosis }(\mathbf{g}) \\
\text { Ingrediente activo/ha }\end{array}$ & $\begin{array}{c}\text { Dosis }(\mathbf{l}+\mathbf{m l}) \\
\text { Material Comercial/ha }\end{array}$ & $\begin{array}{c}\text { Control (\%) } \\
\text { Observado }\end{array}$ \\
\hline Glifosato + Adherente & $1440+240$ & $3,0+300$ & $5,0 \mathrm{a} *$ \\
Glifosato + Extensor & $1440+90$ & $3,0+300$ & $80,0 \mathrm{a}$ \\
Paraquat + Acido Húmico & $1440+72$ & $3,0+600$ & $79,7 \mathrm{a}$ \\
Glifosato + Humectante & $1440+137$ & $3,0+450$ & $78,4 \mathrm{a}$ \\
Paraquat + Extensor & $300+180$ & $1,5+600$ & $70,0 \mathrm{a}$ \\
Paraquat + Humectante & $300+182$ & $1,5+600$ & $69,9 \mathrm{a}$ \\
Glifosato & 1920 & 4,0 & $69,8 \mathrm{a}$ \\
Paraquat + Adherente & $300+480$ & $1,5+600$ & $65,4 \mathrm{a}$ \\
Paraquat + Acido Húmico & $300+90$ & $1,5+750$ & $64,9 \mathrm{a}$ \\
Paraquat & 400 & 2,08 & $0,0 \mathrm{~b}$ \\
TSAH & Testigo & & \\
\hline
\end{tabular}

* Valores con literales desiguales son estadísticamente diferentes (Tukey $1 \%$ ). 
Cuadro 7. Control de malezas en manzano con glifosato y paraquat en mezcla con diferentes surfactantes, 31 DDA en manzano. Arteaga. Coahuila. 1994.

\begin{tabular}{lccc}
\hline $\begin{array}{l}\text { Tratamiento } \\
\text { Nombre técnico }\end{array}$ & $\begin{array}{c}\text { Dosis (g) } \\
\text { Ingrediente activo/ha }\end{array}$ & $\begin{array}{c}\text { Dosis (l+ ml) } \\
\text { Material Comercial/ha }\end{array}$ & $\begin{array}{c}\text { Control (\%) } \\
\text { Observado }\end{array}$ \\
\hline Glifosato + Adherente & $1440+240$ & $3,0+300$ & $100,0 \mathrm{a} *$ \\
Glifosato + Humectante & $1440+137$ & $3,0+450$ & $98,5 \mathrm{a}$ \\
Glifosato + Acido Húmico & $1440+72$ & $3,0+600$ & $98,0 \mathrm{a}$ \\
Glifosato + Extensor & $1440+90$ & $3,0+300$ & $95,3 \mathrm{a}$ \\
Paraquat + Acido Húmico & $300+90$ & $1,5+750$ & $88,4 \mathrm{a}$ \\
Paraquat + Adherente & $300+480$ & $1,5+600$ & $75,1 \mathrm{a}$ \\
Paraquat + Humectante & $300+182$ & $1,5+600$ & $70,9 \mathrm{a}$ \\
Glifosato & 1920 & 4,0 & $69,1 \mathrm{a}$ \\
Paraquat + Extensor & $300+180$ & $1,5+600$ & $65,0 \mathrm{a}$ \\
Paraquat & 400 & 2,0 & $0,0 \mathrm{~b}$ \\
TSAH & Testigo & Testigo & \\
\hline
\end{tabular}

* Valores con literales desiguales son estadísticamente diferentes (Tukey $1 \%$ ).

Durante el período de los 17 y 31 días después de la aplicación, se observó que los síntomas fitotóxicos sobre las malezas variaron de acuerdo al tratamiento; los tratamientos a base de glifosato, presentaron sobre la hierba de golpe (Gaura coccinea) detención del crecimiento apical, apareciendo un rebrote lateral, dando un aspecto de amacollamiento; tallos más largos secos y defoliados en la parte superior y deteniendo la floración. El glifosato sobre correhuela (Ipomoea purpurea) causó una coloración verde oscura, detuvo el crecimiento, no hubo rebrote ni floración. En plántulas de polocotillo (Helianthus laciniatus) con alturas mayores de 23 $\mathrm{cm}$ detuvo el crecimiento, no hubo rebrote, el follaje se secó, los tallos aun estaban verdes pero dañados hasta la raíz. En el quelite cenizo (Chenopodium album) en plantas mayor de $15 \mathrm{~cm}$ detuvo el crecimiento, causó marchitamiento apical. El zacate salvación Bromus unioloides (mayor de $10 \mathrm{~cm}$ ) presentó una coloración amarilla generalizada pero mantuvo cierta suculencia.

En dicho lapso, conforme avanzaron los días, se mantuvieron los síntomas fitotóxicos que presentaron las malezas, se notó un incremento en el control, ya que a los 17 dda el glifosato + adherente mostró un $85 \%$; el glifosato + extensor, $80 \%$; glifosato + ácido húmico, $79,9 \%$ y el glifosato + humectante un $78,4 \%$; mientras que a los 31 DDA los porcentajes de control fueron $100 \% ; 98,5 \% ; 98 \%$ y $95,3 \%$ respectivamente.

En todos los tratamientos con paraquat se observó a los 31 DDA la recuperación (rebrote) de las plantas dañadas en los muestreos anteriores, siguiendo en apariencia con su desarrollo normal.

En el Cuadro 8 se observa que en todos los tratamientos el control disminuyó a los 83 DDA. El gliofo- sato + adherente con un $80 \%$ de control fue considerado como el mejor tratamiento hasta esa fecha. Los tratamientos glifosato + humectante, glifosato + ácido húmico y el glifosato + extensor presentando un 78,6; 77,1 y $75,2 \%$ respectivamente, se consideraron aceptables en control de malezas. El resto de tratamientos fue deficiente en el control a los 83 DDA.

Después de la última observación (83 DDA), las especies sobrevivientes a los tratamientos a base de glifosato y paraquat fueron correhuela, hierba del golpe y polocotillo (mayor de $23 \mathrm{~cm}$ ), las cuales presentaban síntomas fitotóxicos en diferente grado según la mezcla; las malezas tratadas con glifosato + adherente fueron las más dañadas regularmente, su crecimiento se detuvo y no hubo floración, aunque presentaron un ligero rebrote lateral.

Algunas de las características a favor y en contra, así como las limitantes de éstos métodos se deben tener presentes antes de toda actividad sobre las malezas con fines de control.

Algunas de las ventajas que presenta el control químico es que suprime malezas a tiempo, eliminando también a las presentes en hileras, tiene residualidad, no cambia la estructura del suelo y requiere de poca mano de obra; sin embargo, puede afectar a cultivos susceptibles por efecto de acarreo. Las limitantes de su uso están en función del producto a aplicar, tipo de cultivo y maleza, prolongación de la residualidad, su empleo requiere de alto conocimiento técnico.

Por medio del control mecánico se elimina toda la maleza anual, se requiere mínima experiencia, pero no elimina a la maleza en hileras las cuales son considera- 
Cuadro 8. Efecto sobre maleza de los herbicidas postemergentes glifosato y paraquat en mezcla con diferentes surfactantes, 83 DDA en manzano. Arteaga. Coahuila. 1994.

\begin{tabular}{lccc}
\hline $\begin{array}{l}\text { Tratamiento } \\
\text { Nombre técnico }\end{array}$ & $\begin{array}{c}\text { Dosis }(\mathbf{g}) \\
\text { Ingrediente activo/ha }\end{array}$ & $\begin{array}{c}\text { Dosis (I + ml) } \\
\text { Material Comercial/ha }\end{array}$ & $\begin{array}{c}\text { Control (\%) } \\
\text { Observado }\end{array}$ \\
\hline Glifosato + Adherente & $1440+240$ & $3,0+300$ & $80,0 \mathrm{a}^{*}$ \\
Glifosato + Humectante & $1440+137$ & $3,0+450$ & $78,6 \mathrm{a}$ \\
Glifosato + Acido Húmico & $1440+72$ & $3,0+600$ & $77,1 \mathrm{a}$ \\
Glifosato + Extensor & $1440+90$ & $3,0+300$ & $75,2 \mathrm{a}$ \\
Paraquat + Adherente & $300+480$ & $1,5+600$ & $69,0 \mathrm{a}$ \\
Paraquat + Humectante & $300+182$ & $1,5+600$ & $55,1 \mathrm{a}$ \\
Paraquat + Extensor & $300+180$ & $1,5+600$ & $51,1 \mathrm{a}$ \\
Glifosato & 1920 & 4,0 & $50,2 \mathrm{a}$ \\
Paraquat + Acido Húmico & $300+90$ & $1,5+750$ & $49,6 \mathrm{a}$ \\
Paraquat & 400 & 2,0 & $43,1 \mathrm{a}$ \\
TSAH & Testigo & Testigo & $0,0 \mathrm{~b}$ \\
\hline
\end{tabular}

* Valores con literales desiguales son estadisticamente diferentes (Tukey $1 \%$ ).

Cuadro 9. Análisis económico del control químico y mecánico de malezas en Manzano en Arteaga, Coahuila. 1994.

\begin{tabular}{|c|c|c|c|c|c|c|c|c|c|c|}
\hline I Tratamiento & $\begin{array}{c}\text { L o kg mat. } \\
\text { Com./ha } \\
(1+\mathrm{ml})\end{array}$ & aplicac. & $\begin{array}{l}\text { Costo unit. } \\
\text { producto } \\
\text { (l) N\$ }\end{array}$ & $\begin{array}{c}\text { Costo } \\
\text { aplicación } \\
\mathrm{N} \$\end{array}$ & $\begin{array}{c}\text { Costo } \\
\text { supervisor } \\
\mathrm{N} \$\end{array}$ & $\begin{array}{c}\text { Costo total } \\
\text { trat.quím. } \\
\text { N\$ }\end{array}$ & $\begin{array}{c}\mathrm{N}^{\circ} \\
\text { rastreos } \\
\text { por ciclo }\end{array}$ & $\begin{array}{c}\text { Costo } \\
\text { rastreos } \\
\text { /ha N\$ }\end{array}$ & $\begin{array}{c}\text { Costo } \\
\text { supervisor } \\
\text { N\$ }\end{array}$ & $\begin{array}{c}\text { Costo } \\
\text { total trat. } \\
\text { mecánico N\$ }\end{array}$ \\
\hline Glifosato + Adherente & $3,0+300$ & 1 & $58+18,30$ & 230 & 40 & 346,3 & 3 & 230 & 40 & 810 \\
\hline Glifosato + Humectante & $3,0+450$ & 1 & $58+12,10$ & & & 340,1 & & & & \\
\hline Glifosato + Acido Húmico & $3,0+600$ & 1 & $58+15,70$ & & & 343,7 & & & & \\
\hline Glifosato + Extensor & $3,0+300$ & 1 & $58+14,20$ & & & 342,2 & & & & \\
\hline
\end{tabular}

das como más competitivas, no suprime el problema a tiempo, altera la estructura del suelo, además afecta a la raíz; otra limitante es que no controla malezas perennes, al contrario las dispersa, sólo se puede implementar hasta cierta altura del cultivo.

Una vez teniendo más noción de cada método, y de acuerdo al análisis económico se demostró que por medio del control químico se tiene un ahorro del $60 \%$ aproximadamente en comparación con el control mecánico, siendo el costo de N\$345 aproximadamente, mientras que el mecánico es de N\$ 810.

\section{CONCLUSIONES}

Los coadyuvantes (adherente, ácido húmico, humectante y adherente) incrementaron la fitotoxicidad sobre maleza tanto el paraquat como el glifosato.

Los coadyuvantes adherente, ácido húmico y humectante fueron los que más incrementaron la fitotoxicidad del paraquat al menos hasta los 31 DDA.
El adherente, humectante y ácido húmico, incrementaron la fitotoxicidad del glifosato al menos hasta los 83 DDA.

La mezcla de glifosato + adherente $(1440+240 \mathrm{~g}$ i.a./ha) tuvo el valor económico más bajo y un control del $80 \%$ hasta los 83 DDA.

\section{LITERATURA CITADA}

ACADEMIA NACIONAL DE CIENCIAS (NAS). 1990. Plantas nocivas y como combatirlas. Vol. 2 Limusa. México. 574 p.

ARROYO, J. 1980. Revisión bibliográfica de estudios sobre memorias del I Congreso Nacional de la Ciencia de la Maleza. SOMECIMA. Torreón, Coahuila, México. pp 152-169.

BARBERÁ, C. 1976. Pesticidas agrícolas. Omega. España. $569 \mathrm{p}$.

BOLAÑOS, A.; ROSAS, A. 1991. Efecto de cinco surfactantes en la actividad herbicida del glifosato. Memorias 
del XII Congreso Nacional de la Ciencia de la Maleza. SOMECIMA. Acapulco, Guerrero, México. p. 54.

CALDERÓN, E. 1983. Fruticultura general. Limusa. México. $759 \mathrm{p}$.

Catálogo Oficial de Plaguicidas Comisión Intersecretarial para el Control del Proceso y Uso de Plaguicidas, Fertilizantes y Sustancias Tóxicas (CICOPLAFEST); SARH; SEDUE; SS y SECOFI. 1991.. México. 469 p.

DURÁN, S. 1976. Replantación de frutales: sucesión de cultivos y su patología. AEDOS, S.A. Barcelona, España. $332 \mathrm{p}$.

FUENTES, L. 1983. Plagas, enfermedades y malas hierbas. Madrid, España. 115 p.

KLIGMAN, C.; ASHTON, F. 1991. Estudio de las plantas nocivas: principios y prácticas. Limusa. México. 449 p.

KRAMER, S., ACHURICHT,R.; FRIEDRICH, G. 1982. Fruticultura. Continental. México.276 p.

MEDINA, M. 1983. Principios generales sobre herbicidas. In: Curso de plaguicidas agrícolas: generalidades, uso y manejo. Universidad Agraria Antonio Narro. Buenavista, Saltillo, Coahuila. México. pp. 203-206.

MORGADO, L. 1990. Desarrollo de triasulfuron 75 WG y CGA $184927+$ S para el combate de malezas en trigo (Triticum sp.) en México. Series Técnicas de la Asociación Mexicana de la Ciencia de la Maleza. SOMECIMA. 1(1): 23-27.

RAMÍREZ, H.; CEPEDA, M. 1993. El manzano. Trillas S.A. México. 208 p.
REBOUR, H. 1971. Frutales mediterráneos. Mundi-prensa. Madrid, España. 410 p.

ROBERTS, A. 1982. Weed control handbook: Principles. Seventh edition. Blackwell Scientific Publications. USA. 533 p.

ROJAS, M. 1990. Manual teórico práctico de herbicidas y fitorreguladores. Limusa. México. 144 p.

SIERRA, B. 1991. Importancia de las malezas en áreas de explotación agrícola e historia del desarrollo de los herbicidas. In: memorias del curso sobre manejo y control de malas hierbas. SOMECIMA. Acapulgo, Guerrero. México. pp. 1-14.

SOCIEDAD MEXICANA DE LA CIENCIA DE LA MALEZA (SOMECIMA). 1986. Manual de herbicidas. Vol. 1. México. 115 p.

TAMAYO, M. 1990a. Absorción y translocación de los herbicidas. In: memorias del IX Congreso Nacional de la Ciencia de la Maleza. SOMECIMA. Escuela de Agricultura hermanos Escobar. Ciudad Juárez, Chihuahua. México. p 61-81.

TAMAYO, M. 1990b. Problemas de la maleza y su manejo integrado en trigo para el norte de México. Series técnicas de la Asociación Mexicana de las Ciencia de la Maleza, México. SOMECIMA 1(1): 3-11.

URZÚA, F.; SÁNCHEZ, A. 1991. Surfactantes en la acción biológica de herbicidas post-emergentes en invernadero y campo. In: memorias de XII Congreso Nacional de la Ciencia de la Maleza. SOMECIMA. Acapulco, Guerrero. México. p 58.

YOUNG, S. 1984. Glyphosate plus adjuvants. United States of America. Horticultural abstracs. 54 (6): 305. 